\title{
Guía de intervenciones enfermeras pre y post hemodiálisis para unidades de hospitalización
}

\author{
Cristina Valiño Pazos - Mª Jesús Pérez Paz - Modesta Mojón Barcia
}

Complexo Hospitalario Universitario Juan Canalejo. A Coruña)

\section{Sra. Directora:}

Siendo la enfermería una ciencia multidisciplinar y nuestro trabajo diario una actividad multitarea que abarca infinidad de patologías, y diversidad de pacientes, no es extraño que en nuestra profesión, a veces, manejemos los conocimientos imprescindibles y no los deseables $y$, en ocasiones, no apliquemos los cuidados enfermeros con criterios de calidad total.

En nuestra unidad de agudos, recibimos diariamente los pacientes que, ingresados en el complejo hospitalario, son remitidos desde las diferentes unidades de hospitalización para su tratamiento de hemodiálisis. Tras observar que los pacientes no llegan en muchas ocasiones en las condiciones ideales desde el punto de vista de los cuidados de enfermería, comprobamos que la mala praxis está más relacionada con un desconocimiento del enfermo renal y de la terapia de hemodiálisis que con una falta de tiempo, medios, interés o profesionalidad. Por otra parte, resulta significativo que los pacientes acudan mejor preparados si su ingreso es en la unidad de nefrología que si, por el contrario, están ubicados en una unidad no especializada en el paciente renal ya que la enfermería de ne-

\begin{tabular}{|c|}
\hline Correspondencia: \\
Modesta Mojón Barcia \\
Unidad Diálisis \\
Hospital Juan Canalejo \\
Xubias de Arriba, 84 \\
15006 A Coruña \\
crisvalin@yahoo.es \\
\hline
\end{tabular}

frología conoce más profundamente al enfermo renal y su singular manejo por lo que decidimos buscar el modo de proporcionar la información imprescindible para que el cuidado óptimo de nuestros pacientes no dependiese de un factor tan arbitrario como su ubicación hospitalaria.

Decidimos diseñar un soporte educativo, sencillo y eficaz, que transmita, a los profesionales enfermeros, la información imprescindible para garantizar una atención integral, en cualquier planta de hospitalización, al paciente que necesita tratamiento de hemodiálisis.

Para ello, hicimos una recopilación de las deficiencias observadas en la prestación de cuidados enfermeros al paciente sometido a hemodiálisis e ingresado en unidades periféricas al servicio de nefrología. Analizamos los datos obtenidos a través de métodos cualitativos: grupo nominal y observación participante.

Posteriormente, definimos los principales cuidados a promover y las actividades de enfermería necesarias para lograrlos y finalmente diseñamos un material educativo que se remitirá a las plantas de hospitalización y cumplirá los siguientes requisitos: fácil acceso, sencillo, concreto, motivador, atractivo...

Tras la observación diaria de los pacientes en nuestra área de agudos enumeramos como más frecuentes las siguientes deficiencias en su manejo terapéutico: 
- Administración previa a hemodiálisis de farmacoterapia que sufre degradación intradiálisis, disminuyendo por tanto sus niveles séricos y perdiendo actividad al mantener valores por debajo del rango terapéutico.

- Manejo inadecuado en la aplicación de anestésicos locales repercutiendo negativamente en el confort del paciente, que como consecuencia se somete a la canalización de los accesos vasculares para la hemodiálisis sin la anestesia local/analgesia pautada.

- Permanencia superior a la recomendada de los apósitos hemostáticos con riesgo de alteración de la integridad cutánea (maceración, irritación, desarrollo de hipersensibilidad 0 alergias, ...)

- Duplicación de la dosis de anticoagulante por aplicación de terapia subcutánea que se suma a la administración intradiálisis de la forma intravenosa, aumentando el riesgo de complicaciones por alteración de la coagulación.

- Manipulación incorrecta de las vías centrales e inadecuada heparinización.

- Desconocimiento de los cuidados de la FAV así como de la resolución de sus principales complicaciones.

- Déficit de conocimientos dietéticos para proporcionar información y resolver dudas al paciente.

A partir de los puntos expuestos se definen las principales áreas para la mejora de los cuidados y se diseña un cartel informativo (figura 1) que se distribuye en todas las unidades de hospitalización susceptibles de acoger pacientes nefrológicos. Dicho cartel representa un cuerpo humano en posición anatómica y contiene de modo esquemático los diferentes cuidados que requiere tanto para afrontar la hemodiálisis en las mejores condiciones posibles como para recuperar su equilibrio bio-psico-social a la mayor brevedad tras el tratamiento. Dicho cartel se expondrá en lugar bien visible y accesible para todo el personal, de modo que su consulta sea posible de modo cómodo y rápido. De este modo cumplirá una misión orientativa del personal de enfermería tanto para la recepción del paciente renal como para el establecimiento de prioridades, sirviendo de guía para el diseño del plan de cuidados en todas sus etapas de valoración, planificación, ejecución y evaluación continua.
Este primer trabajo, forma parte de un proyecto más amplio que desarrollaremos en un futuro próximo y que pretende mejorar la formación con respecto al paciente renal de los profesionales de nuestro complejo hospitalario. Hemos querido comenzar con esta pequeña "campaña educativa" porque su atractivo intentará conquistar la curiosidad y el interés de nuestros compañeros. $Y$ a partir de este primer acercamiento intentaremos promover la colaboración interprofesional que tanto beneficia a nuestra actividad enfermera. En sucesivos estudios analizaremos la acogida de nuestra iniciativa, así como su repercusión y utilidad, porque resulta imprescindible para la atención especializada de un usuario cada vez más exigente conocer en profundidad su realidad como paciente, porque los cuidados enfermeros de calidad han de sostenerse en una firme base de conocimientos científicos, porque mientras sólo nos concedan siete especialidades enfermeras nosotras mismas debemos buscar nuestra especialización y porque mejor que nadie sabemos que no existe peor castigo para el experto enfermo renal que sospecharse en inexpertas manos.

\section{Bibliografía}

1. Andrés J, Fortuny C. Cuidados de enfermería en la insuficiencia renal. Madrid: Gallery/HealthCom; 1993.

2. Andreu L, Force E. 500 cuestiones que plantea el cuidado del enfermo renal. Barcelona: Masson; 2001.

3. Botella J. Manual de nefrología clínica. Barcelona: Masson; 2002.

4. García AI. Formación a enfermería de hospitalización en relación al cuidado del paciente en tratamiento dialítico. En: Libro de Comunicaciones XXX Congreso Sociedad Española de Enfermería Nefrológica; Palma de Mallorca 15-18 0ctubre del 2003.

5. www.seden.org

6. www.insuficienciarenalcrónica.com 


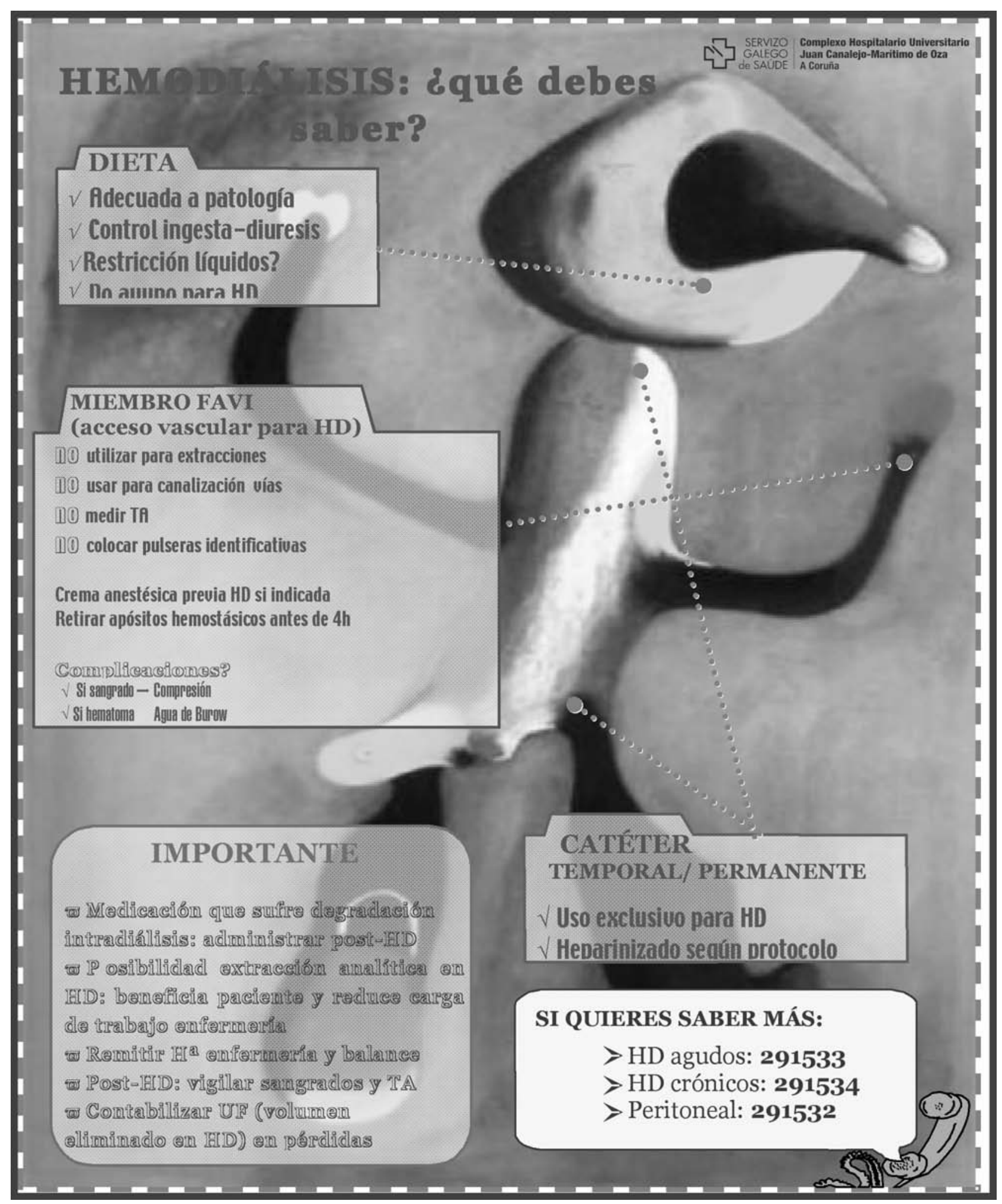

Figura 1: Guía de intervenciones enfermeras pre y post hemodiálisis para unidades de hospitalización 